\title{
Infección intrahospitalaria por Klesbsiella Pneumoniae en un brote de sepsis en recién nacidos.
}

\author{
Drs. Guerrero Ode, P.; Francotte, N.; Pinc, M.; Vaisman, S.**
}

\begin{abstract}
ABSTHACT
1. Clinical and bacteriological studies were performed in 17 newborn babies with Klebsiella pneumoniae sepsis between April and September 1978.

2. The onset of the disease was 7.5 days of life as a mean. Premature rupture of membranes we lound in 4 newborn $(23 \%)$. No relation was found between infections during pregnancy and newbon sepsis; $53 \%$ of the newborm were delivened by cesarean section.

3. $53 \%$ of the newborn were preterm babies, $70 \%$ had a birh weight less than $2.500 \mathrm{grams}$. The Apgar score at one ninute was equal or less thun 5 in $47 \%$ of the newborn.

4. The physical examination showed hepathomegaly in $47 \%$, pallor in $70 \%$, $33 \%$ were hypoactive, $41 \%$ dehidrated and with jaundice and $35 \%$ presented upneic episodes.

5. Klebsiella pneumoniae was found in cultures from personnel and environment.

6. All the strains were studied in their antibiotic susceptibility. These tindings revealed 3 groups clearly defined.

7. Mortality rate found was $41 \%$ in the affected group.
\end{abstract}

La infección intrahospitalaria es actualmente uno de los problemas frecuentes con que se enfrenta el clínico, situación que es importante por;

a) aparición de cepas multirresistentes a los antibióticos,

b) las cepas hospitalarias pueden provocar infecciones con carácter endémico o de brote epidémico,

c) dificultades para cortar la cadena de portación, colonización e infección.

Los Servicios de Recién Nacidos presentan un alto riesgo de infección intrahospitalaria en porcentajes que van de un 15 a un $25 \%$ de acuerdo a la literatura extranjera. Actualmente los bacilos Gram (-) son los mayores patógenos involucrados y son los responsables del $70 \%$ o más de estas infecciones en dichos Servicios. ${ }^{1.2}$

Ocasionalmente estas infecciones pueden ser transmitidas por un miembro del equipo médico que aloja a estos agentes en sus manos, ${ }^{3}$ vagina o recto. ${ }^{4}$ Lo más común es que las manos sean un mero vehiculo de transmisión de la bacteria de un niño a otro y los reservorios sean los mismos pacientes. $^{5}$

Aunque el desarrollo de las unidades de cuidado intensivo neonatal reduce la mortalidad de los

-Depurtamento de Medicina Experimental, Fac. de Medicina Sur, Universidad de Chile.

**Departamento de Clínjcas, Facultad de Medicina Sur, Universidad de Chile.

Maternidad del Hospital Barros Luco. recién nacidos con patología grave, también introduce una serie de instrumentaciones y técnicas propias del progreso en el diagnóstico y tratamiento $y$ con ello se aumenta el riesgo de infecciones. $6,7,8,9$

La mayoria de los informes destacan a Klebsiella pneumoniae con una incidencia de $25 \%$ como agente etiológico de sepsis en el recién nacido ${ }^{10}$. 11,12 y con un alto grado de mortalidad. El amplio uso de la terapia antibiótica a menudo lleva a la selección y predominio dè organismos multirresistentes dentro de estas especies, así como de otras. $13,14,15,16$

El presente trabajo describe un brote de sepsis a Klebsiella pneumoniae, con sus características clínicas, bacteriológicas y epidemiológicas en recién nacidos del Servicio de Neonatología de la Maternidad del Hospital Barros Luco - Trudeau, en el período abril a septiembre de 1978.

\section{MATERIAL Y METODO}

Se estudiaron 17 recién nacidos desde el punto de vista clínico y bacteriológico, que presentaban signos clinicos de sepsis entre abril y septiembre de 1978 y cuyo agente aislado en todos los casos fue Klebsiella pneumoniae.

Se consideró diagnóstico presuntivo de sepsis, la aparición de los siguientes signos y síntomas: ictericia, intolerancia alimentaria, compromiso respiratorio y/o gastrointestinal, fiebre, convul- 
siones, hipoactividad, hepatomegalia, esplenomegalia. A todos ellos se les exigió como diagnóstico de certeza el aislamiento del agente etiologi co, en el hemocultivo y/o urocultivo y $/ 0$ cultivo de líquido cefalorraquídeo. Se consignaron los principales datos clinicos del niño y de la madre, junto con su evolución, tratamiento y estudio bacteriológico.

Para el aislamiento del agente etiológic s se tomaron muestras de sangre, de líquido cefalorraquideo y de orina, esta última siempre por punción vesical. La sangre se obtuvo por punción venosa extravéndose $1 \mathrm{ml}$., la cual fue sembrada inmediatamente en tubos con $20 \mathrm{ml}$. de caldo tripticase y se incubó a $35^{\circ} \mathrm{C}$. Se observaron desde las 18 horas de incubación hasta los 7 días. ${ }^{17} \mathrm{El}$ líquido cefalorraquideo y la orina se sembraron de acuerdo a la metodología habitual.

Los hemocultivos que presentaban probable desarrollo bacteriano se resembraron en placas de agar sangre y agar Mac Conkey y posteriormente si eran bacilos Gram (-) se procedio a identificarlos por estudio de sus propiedades bioquímicas de acuerdo a método estandar. ${ }^{18}$ Igual identificación se realizó a partir del cultivo primarin de L.C.R. y de orina.

Pruebas bioquímicas efectuadas: gluconato de potasio, inositol, arabinosa, gas de glucosa, lactosa, indol, Voges Proskauer, rojo de metilo, citrato de Simmons, ureasa, adonitol, H2S, licuación de gelatina, dulcitol, manitol, sacarosa, sorbitol, rafìnosa, ramnosa, malonato, decarboxiliación de lisina y ornitina.

Además del estudio de muestras clínicas se hizo control bacteriológico del personal y ambiente de la unidad. A un total de 110 personas (69 auxiliares, 24 matronas y 17 médicos) que trabajan en la unidad, se les tomó secreción faríngea y muestras de manos en dos oportunidades separadas por un tiempo de 35 días. La secreción faríngea se tomó con tórula y se sembró inmediatamente en placas de agar citrato de Simmons y la muestra de manos se tonó por impresión de los dedos sobre la super. ficie de utras placas que contenian el mismo medio de cultivo. Todas las placas se incubaron por 24 a 48 horas a $35^{\circ} \mathrm{C}$. El control bacteriológico de] ambiente dirigido a la buísqueda de Klebsiella se realizó en 58 muestras: aire, ropa de cama, ropa del personal. superficie de mesones, lavatorios. Las inuestras fuerorr tomadas de acuerdo a métodos habituales, utilizando nuevamente el medio de Simmons. Todas las colonjas sospechosas de
Klebsieila fueron estudiadas igual que las cepas aisladas en los enfermos.

A todas las cepas identificadas como Klebsiella pneumoniae, provenientes de los enfermos, el personal y el ambiente se les practicó antibiograma por difusión en agar de acuerdo al método de Kirby-Bauer. ${ }^{19}$ Se estudió su comportamiento frente a los siguientes antibióticos: ampicilina, carbenicilina, tetraciclina, kanamicina, gentamicina, cloramfenicol, rifamicina, sulfametoxazol-trimetoprim, cefalosporina y sulfato de colis* tin. Se usaron tabletas Neo-Sensitabs A/S Rosco Denmark.*

\section{RESULTADOS}

En el eśtudio bacteriológico de muestras tomadas en 17 recién nacidos con diagnóstico clínico de sepsis en el Servicio de Neonatología del Hospital Barros-Luco-Trudeau, se aislaron is cepas: 15 en sangre, 2 en liquido cefalorraquídeo y una en orina. Todas ellas tuvieron un comportamiento bioquímico correspondiente a Klebsiella pneumoniae: glucosa ácida con gas, lactosa ácida con gas, indol $(-)$, Voges Proskauer $(+)$, rojo metilo $(-)$, citrato de Simnons $(+)$, ureasa $(+)$, malonato $(+)$, H2S (-), gelatina (-), decarboxilación de lisina $(+)$, y ornitina $(-)$, inositol $(+)$, dulcita $(+) 0$ $(-)$, gluconato de potasio $(+)$, adonita $(+)$, manitol $(+)$, sacarosa $(+)$, sorbitol $(+)$, arabinosa $(+)$, rafinosa $(+)$, ramnosa $(+)$, manosa $(+)$.

En la tabla N. ${ }^{0} 1$ se presenta la distribución en el tiempo de los pacientes, el tipo de muestra $y$ la fecha en que fue aislado el agente y los dias de vida del recién nacido en relación con la iniciación del cuadro clínico.

En ella se destaca que el $53 \%$ de los casos se presentaron en la última semana de agnsto y primeros 2 dias de septiembre de 1978. El promedio de inicio de la enfermedad fue de 7,5 dias de vida con rango de 2 y 28 días. El agente etiológico se aisló en algunos casos además de los consignados en coprocultivos (4), secreción ocular (1) y pus proveniente de abcesos (4).

La Tabla 2 presenta los antecedentes matemios. La edad de la madre va descle los 15 a los 40 años (promedio 24,30). En 4 casos existe el antecedente de rotura prematura ovular. El comienzo del chadro séptico en los recién nacidos de estas ma-

*Gentileza de DIMY L Ltda. 
dres fue entre los 2 y 10 días de vida (promedio 6,75).

En 12 de las 17 madres no hay antecedentes de infección; en las restantes, 2 de ellas, los antecedentes no están relacionados con la sepsis a Klebsiella del recién nacido, como es el caso de una fiebre tifoidea y una serologia positiva para sífilis. En los 3 casos restantes una es portadora de una pielonefritis crónica, otra con infección ovular y la última portadora de un síndrome febril. Con respecto al tipo de parto, el $53 \%$ de los recién nacidos nacieron por cesárea.

\section{TABLA 1}

Aislamiento de Klebsiella pneumoniae en sepsis del recién nacido entre el 8 de abril al 2 de septiembre de 1978. Maternidad Hospital Barros Luco.

\begin{tabular}{|c|c|c|c|c|c|}
\hline R.N. & HEMO & L.C.R. & URO & DIAS DE VIDA & FECHA CULTIVO \\
\hline EMV & & & 1 & 12 & $8 / 4$ \\
\hline MVL & 1 & & & 10 & $20 / 4$ \\
\hline I R T & 1 & & & 5 & $24 / 4$ \\
\hline$M M N$ & 1 & & & 10 & $31 / 5$ \\
\hline MVD & 1 & & & 6 & $2 / 6$ \\
\hline S B CH & 1 & & & 8 & $23 / 6$ \\
\hline VAV & 1 & 1 & & 2 & $26 / 6$ \\
\hline$G \vee Q$ & 1 & & & 15 & $2 / 7$ \\
\hline F P R & 1 & & & 5 & $28 / 8$ \\
\hline J S T & & 1 & & 3 & $28 / 8$ \\
\hline$M F V$ & 1 & & & 3 & $28 / 8$ \\
\hline R D T & $\mathbf{l}$ & & & 7 & $30 / 8$ \\
\hline E CH V & 1 & & & 28 & $30 / 8$ \\
\hline PVR & 1 & & & 3 & $30 / 8$ \\
\hline MR R & 1 & & & 5 & $1 / 9$ \\
\hline Z B J & 1 & & & 4 & $1 / 9$ \\
\hline E S B & 1 & & & 2 & $2 / 9$ \\
\hline
\end{tabular}

Tabla 2

Antecedentes de las madres de 17 R.N. con sepsis a Klebsiella pneumoniae. Matemidad Hospital Barros Luco. Abril-septiembre, 1978

\begin{tabular}{lclll}
\hline MADRE & EDAD & \multicolumn{1}{c}{ PATOLOGIA } & INFECCION & \multicolumn{1}{c}{ PARTO } \\
\hline E.M.V. & 22 & T de PP & FEBRIL & ESP. \\
M.V.L. & 23 & RPO (48) & & CS \\
I.R.T. & 23 & PRE ECLAM & & FP \\
M.M.N. & 24 & RPO(23) & & CS \\
M.V.D. & 15 & T de PP & & POD \\
S.B.CH. & 21 & PP & & POD \\
V.A.V. & 19 & & & CS \\
G.V.Q. & 21 & & & ESP \\
F.P.R. & 40 & CIE & TIFOIDEA & ES \\
J.S.T. & 29 & & & ESP \\
M.F.V. & 26 & & VDRL+ & CS \\
R.D.T. & 35 & PRE ECLAM & PIELONEFRITIS & CS \\
E.CH.V. & 37 & HIPERTENSION & & ESP \\
P.V.F. & 21 & & INF OVUL & CS \\
M.R.R. & 18 & RPO (47) PROC CORDON & & CS \\
Z.B.J. & 21 & VICIO PELVICO & & CS \\
E.S.B. & 18 & R PO (B) & & \\
\end{tabular}

*En paréntesis el número de horas de rotura ovular. 
La Tabla 3 presenta los antecedentes de los recién nacidos estudiados incluyendo peso de nacimiento, test de Apgar al minuto, 5 y 10 minutos, distribución por sexo, edad gestacional y diagnóstico presuntivo al ingreso.

Peso de nacimiento $y$ edad gestacional: $12 \mathrm{de}$ los recién nacidos tienen peso menor de $2.500 \mathrm{~g}$. En este grupo hay 3 que son PEG y de los 9 restantes, 6 pesaron al nacer menos de $1.500 \mathrm{~g}$. Observando el grupo total, se destaca la alta incidencia de la infección a Klebsiella en recién nacidos de bajo peso de nacimiento.
Un $47 \%$ de los niños presentaron scores de Apgar igual o menor a 5, revelando cierto compromiso hipoxico.

En relación al sexo, un $65 \%$ de los reciến nacidos fueron de sexo masculino.

En la Tabla 4 aparecen los signos clínicos más frecuentemente observados en los 17 recién nacidos con sepsis a Klebsiella. Pretominan la hepatomegalia, con un $75 \%$; la palidez terrosa, $70 \%$; hipoactividad, $53 \%$; deshidratación, $41 \%$, e ictericia, $41 \%$.

\section{Tabla $\mathbf{3}$}

Antecedentes de 17 R.N. can sepsis a Klebsiella. Matemidad Barros Luco. Abril-septiembre, 1978

\begin{tabular}{|c|c|c|c|c|c|}
\hline MADRE & PESO & APGAR & SEXO & DIAGNOSTICO & SEM. GEST \\
\hline E.M.V. & 1.120 & $1-3-7$ & $\mathbf{F}$ & HIPOX.gr & 32 \\
\hline M.V.L. & 1.410 & $7-8-8$ & $\mathbf{M}$ & SDRI-RPO & 30 \\
\hline I.R.T. & 2.350 & 8-9 & $\mathrm{F}$ & PEG & 40 \\
\hline M.M.N. & 1.150 & 6-8 & $\mathbf{M}$ & SDR-RPO & 33 \\
\hline M.V.D. & 1.750 & $4-7-7$ & $\mathbf{M}$ & SDRI & 33 \\
\hline S.B.CH. & 1.400 & $6 \cdot 8 \cdot 9$ & $\mathbf{M}$ & & $31-32$ \\
\hline V.A.V. & 2.500 & $1-4-7$ & $\mathbf{M}$ & HIPOX gr PEG & 40 \\
\hline G.V.Q. & 3.540 & $8-9$ & $\mathbf{M}$ & CARDIP CONG & 39 \\
\hline F.P.R. & 2.640 & $5-8-9$ & $\mathbf{F}$ & & 40 \\
\hline J.S.T. & 3.440 & $2-5-7$ & $\mathbf{M}$ & HIPOX gr HIPOGL] & 40 \\
\hline M.F.V. & 1.800 & $1-7-8$ & $\mathbf{M}$ & HIPOX gr PEG & 40 \\
\hline R.D.T. & 1.800 & $5 \cdot 7-9$ & $\mathbf{M}$ & HIPOX gx & 36 \\
\hline E.CH.V. & 1.440 & 8-9 & $\mathrm{F}$ & PEG & 35 \\
\hline P.V.R. & 3.420 & $8-9$ & $\mathbf{M}$ & & 38 \\
\hline M.R.R. & 1.300 & $2-4-8$ & $\mathrm{~F}$ & HIPOX gr RPO SDRI & 32 \\
\hline Z.B.J. & 2.690 & $1-6-8$ & $\mathrm{~F}$ & HIPOX gr SDR ASP & 40 \\
\hline E.S.B. & 1.905 & $8-9$ & $\mathbf{M}$ & RPOSDRI & 33 \\
\hline
\end{tabular}

Tabla 4

Incidencia de los principales signos clinicos en 17 R.N. con sepsis a Klebsiella pneumoniae. Matemidad Hospítal Barros Luco. Abril-septiembre, 1978

\begin{tabular}{|c|c|c|c|c|c|}
\hline SIGNOS & No & $\%$ & SIGNOS & $x 0^{\circ}$ & $\%$ \\
\hline Hepatomegalia & 13 & 75 & Polipnea & 4 & 24 \\
\hline Paliḍez terrosa & 12 & 70 & Hiporteflexia & 4 & 24 \\
\hline Hipoactividad & 9 & 53 & Fiebre & 4 & 24 \\
\hline Deshidratación & 7 & 41 & Irritabilidad & 4 & 24 \\
\hline Ictericia & 7 & 41 & Convulsiones & 3 & 18 \\
\hline Apnea & 6 & 35 & Esplenomegalia & 3 & 18 \\
\hline Bronconeumonía & 5 & 30 & Vómitos & 2 & 12 \\
\hline
\end{tabular}


Aislamiento de Klebsiella en el personal y ambiente inanimado. Matemidad Hospital Barros Luco. Abril-septiembre, 1978

En paréntesis el número de cultivos positivos a Klebsiella. $\mathrm{N}$ = número de muestra,

Total personal: 220

MUESTRAS PERSONAL
Tokal ambiente: $\mathbf{5 8}$ muestras

\section{MUESTRAS AMBIENTE}

\begin{tabular}{|c|c|c|c|c|c|}
\hline \multirow{2}{*}{$\frac{\text { Personal }}{\text { Auxiliares }}$} & \multicolumn{2}{|c|}{ Manos } & \multicolumn{2}{|c|}{ Faringe } & \multirow{2}{*}{$\begin{array}{l}\text { Lavamanos (4), llaves agua, delantales (1), fonendos- } \\
\text { copios, incubadoras, puertas, manillas, cajas instru- } \\
\text { mental, sabanillas, resucitador, refrigerador, cunas. }\end{array}$} \\
\hline & 69 & (6) & 69 & (1) & \\
\hline Matronas & 24 & $(0)$ & 24 & (0) & \\
\hline Médicos & 17 & (0) & 17 & (0) & $\mathrm{N}^{\circ}=\mathbf{5 8}$ \\
\hline Total & 110 & & 10 & & \\
\hline
\end{tabular}

Tabla 6

Relación y evolución en el tiempo de los patrones de resistencia y sensibilidad de las cepas de Klebsiella disladas en los recién nacidos con sepsis

\begin{tabular}{|c|c|c|c|c|}
\hline Patrones de resistencia & Sensibilidad & N. patrón & N.omuestris & Fecha \\
\hline CB, AM, CAF, GE, RIF. & $\begin{array}{l}\text { KANA, TE, COLIS, } \\
\text { CEFALOS, SULFA- } \\
\text { TRIM. }\end{array}$ & 1 & $\begin{array}{l}2 \text { HENO } \\
\text { I URO }\end{array}$ & $8 \mathrm{al} \mathrm{24/4}$ \\
\hline $\begin{array}{l}\text { CB, AN, CAF, GE, RIF, } \\
\text { TE, KANA. }\end{array}$ & $\begin{array}{l}\text { COLIS, CEFALOS, } \\
\text { SULFA-TRIM. }\end{array}$ & 2 & 2 HEMO & $31 / 5$ al $2 j 6$ \\
\hline $\begin{array}{l}\text { CB. AM, CAF, GE, KANA, } \\
\text { RIF, TE, SULFA-TRIM. }\end{array}$ & COLIS, CEFALOS. & 3 & $\begin{array}{l}2 \text { HEMO } \\
1 \text { LCR }\end{array}$ & 23 al $26 / 6$ \\
\hline $\begin{array}{l}\text { CB, AN, CAF, GE, KANA, } \\
\text { TE, RIF. }\end{array}$ & $\begin{array}{l}\text { COLIS, CEFALOS, } \\
\text { SULFA-TRIM. }\end{array}$ & 2 & $\begin{array}{l}9 \text { HEMO } \\
\text { I LCR }\end{array}$ & $2 / 7$ al $2 / 9$ \\
\hline
\end{tabular}

$\begin{array}{llll}\text { AMI } & =\text { Ampicilina } & \text { GE } & =\text { Gentamicina } \\ \text { CAF } & =\text { Clonimfenicol } & \text { KANA } & =\text { Kanamicina } \\ \text { CB } & =\text { Carbenicilina } & \text { TE } & =\text { Tetraciclina } \\ \text { COLIS } & \text { Sulfato de Colistin } & \text { SULFA-TRIM } & =\text { SuIfametoxazol-trimetoprim } \\ \text { CEFALOS } & =\text { Cefalosporina } & \text { RIF } & =\text { Fifamicina }\end{array}$

En la Tabla 5 se resume el resultado de los cultivos efectuados en manos y faringe del personal de la Unidad de Recién Nacidos y en las muestras del medio ambiente.

De 110 muestras tomadas en las manos del personal se obtuvo cultivo positivo a Klebsiella en 6 y el único cultivo faringeo positivo de los 110 realizados correspondió a una de estas personas.

De las 58 muestras tomadas en distintas partes del ambiente, se aisló Klebsiella en 4 muestras tomadas de lavamanos y una proveniente de un delantal. En el segundo control bacteriológico del personal, realizado 35 días después, no se aisló
Klebsiella, sin mediar ningún tratamiento en el intervalo.

La Tabla 6 presenta los patrones de resistencia a los antibióticos de las cepas estudiadas y su evolución en el tiempo y la sensibilidad encontrada. Frente a los antibióticos estudiados se observaron 3 comportamientos diferentes en relación a los patrones de resistencia. El patrón asignado con el número 2 apareció el 31 de mayo al 2 de junio, para reaparecer el 2 de julio al 2 de septiembre de 1978. Las cepas aisladas en el personal y ambiente se comportaron con el patrón de resistencia asignado en el número 2. 
En la Tabla 7 se presenta el total de las cepas de Klebsiella aisladas tanto en pacientes como personal y ambiente en relación con los patrones de resistencia observados. El $80 \%$ correspondió a cepas que tenían un comportamiento de resistencia similar, encasillándose en el patrón número dos.

Tabla 7

Porcentaje de cepas de Klebsiella de acuerdo a los diferentes patrones de resistencia asignados. Matemidad Hospital Barros Luco. Abril-septiembre, 1978

\begin{tabular}{lccc}
\multicolumn{1}{c}{ Fuente } & \multicolumn{3}{c}{ Patrón de resistencia } \\
\hline \multicolumn{1}{c}{ Sangre } & 1 & 2 & 3 \\
LC.K. & 2 & 11 & 2 \\
Orina & - & 1 & 1 \\
Ambiente & 1 & - & - \\
Personit & - & 5 & - \\
\hline Total & $3=10 \%$ & $24=80 \%$ & $3=10 \%$ \\
\hline
\end{tabular}

En la Tabla 8 se presenta la mortalidad de los recién nacidos en relación al peso de nacimiento $y$ test de Apgar.

La mortalidad fue de $7(41 \%)$ recién nacidos, 5 de los cuales tuvieron un peso menor de $2.500 \mathrm{~g} \mathrm{al}$ nacer. No hubo relación significativa entre el test de Apgar y la mortalidad.

\section{Tabla 8}

Relación entre mortalidad, peso de nacimiento $y$ test de Apyar en 17 R.N. con sepsis a Klebsiella. Hospital Barros Luco. Abril-septiembre, 1978

\begin{tabular}{llll}
\hline R.N. & Peso & Apgar 1'5'y $10^{\prime}$ & Mortalidad \\
\hline E.M.V. & 1.120 & $1-3-7$ & $\mathrm{~V}$ \\
M.V.L. & 1.410 & $7-8-9$ & $\mathrm{~F}$ \\
I.R.T. & 2.350 & $8-9$ & $\mathrm{~V}$ \\
M.N.V. & 1.150 & $6-8$ & $\mathrm{~V}$ \\
M.V.D. & 1.750 & $4-7-7$ & $\mathrm{~F}$ \\
S.B.C.H. & 1.400 & $6-8-8$ & $\mathrm{~V}$ \\
V.A.V. & 2.500 & $1-4-7$ & $\mathrm{~V}$ \\
G.V.Q. & 3.540 & $8-9$ & $\mathrm{~F}$ \\
F.P.R. & 2.640 & $5-8-9$ & $\mathrm{~V}$ \\
J.S.T. & 3.440 & $2-5-7$ & $\mathrm{~V}$ \\
M.F.V. & 1.800 & $1-7-8$ & $\mathrm{~F}$ \\
R.D.T. & 1.800 & $5-7-9$ & $\mathrm{~V}$ \\
E.CH.V. & 1.440 & $8-9$ & $\mathrm{~V}$ \\
P.V.R. & 3.420 & $8-9$ & $\mathrm{~V}$ \\
M.R.R. & 1.300 & $2-4-8$ & $\mathrm{~F}$ \\
Z.B.J. & 2.690 & $1-6-8$ & $\mathrm{~F}$ \\
E.S.B. & 1.905 & $8-9$ & $\mathrm{~F}$ \\
\hline
\end{tabular}

$\mathbf{v}=$ rivo

$F=$ fallecido

\section{DISCUSION}

La infección intrahospitalaria es un problema actual y común que afecta en distinto grado y trascendencia a los pacientes hospitalizados.

En la actualidad el problema se agrava, porque los agentes bacterianos involucrados pueden presentar multirresistencia a los antibióticos de uso clínico.

La infección intrahospitalaria en los Servicios de R.N. se favorece porque los pacientes expuestos presentan factores predisponentes como: inmadurez, bajo peso, hipoxia, etc., $y$ además están sujetos a manipulaciones diagnósticas y/o terapéuticas. $^{\text {' }}$

Estas infecciones adquieren trascendencia debido a la facilidad con que los agentes infectantes invaden los tejidos del $\mathbf{F} . \mathbf{N}$, originando graves cuadros de sepsis. 27

Durante el transcurso de un programa de investigación de sepsis del R.N. en el Servicio de Neonatología de la Matemidad del Hospital Barros Luco Trudeau se detectó un brote de septicemias entre abril $y$ septiembre de 1978. El agente etiológico fue aislado en 17 pacientes e identificado como Klebsiella pneumoniae, multirresistente a antibióticos.

Los dias previos de vida y de hospitalización al inicio del cuadro séptico estuvieron en un rango de 2 a 29 días (promedio 7.5), hecho que está a favor de la posibilidad de que este agente provenga del ambiente hospitalario. Aunque algunos R.N. podrian haber adquirido la cepa perinatalmente de la flora materna, lo cual no podemos descartarlo, ya que ella no se investigó, el hecho de que el $53 \%$ de estos R.N. nacieron por cesárea disminuye esta posibilidad. ${ }^{21}$

Además se presentó un aumento del brote en un período de 6 días entre el $28 / 8$ y $2 / 9$, constituyendo el $53 \%$ del total de los casos. En todos ellos con el aislamiento de Klebsiella pneumoniae multirresistente.

Cuatro de los R.N. estudiados provenían de un embarazo con rotura precoz de membrana con más de 20 horas de rotura. Estos niños iniciaron el cuadro séptico entre los 2 y 10 días de vida, con un promedio de 6.7 dias, el cual no se aleja del promedio general, que es de 7.5 días. Esto apoya la presunción de haber adquirido la infección inde. pendientemente de RPO, pues si la infección ovular hubiera sido el foco de contaminación para estos R.N., la aparición de la infección hubiera 
sido más precoz y se esperaría encontrar septicemias con diversos agentes etiológicos y no el mismo agente en todos ellos.

Klebsiella pneumoniae se aisló no sólo en el hemocultivo, LCR o urocultivo de los R.N., también se aisló en manos de una enfermera y dos veces en el lavatorio de la Unidad de Tratamiento Intensivo. Al analizar los resultados bacteriologicos buscando marcadores epidemiológicos de infección intrahospitalaria por Klebsiella pneumoniae, solamente pudimos investigar y relacionar los diferentes biotipos encontrados, los cuales nos presentan un solo biotipo predominante y al analizar la resistencia a antibióticos presentada por las diferentes cepas, nos encontramos que uno de los patrones asignados arbitrariamente con el N. ${ }^{\circ} 2$ (Tabla 6) es el más frecuente y el único que se detectó en las cepas aisladas en las manos del personal y ambiente, lo cual está de acuerdo con lo demostrado por otros autores ${ }^{20,25}$ para asegurar que la infección es realmente intrahospitalaria.

E] origen o aparición de la multirresistencia pudiera ser plasmidial y estar seleccionado por la exposición de la Klebsiella pneumoniae a los antibióticos que se usan en el Servicio, con un mecanismo de esparcimiento de su reservorio (lavamanos obstruido), a las manos del personal e instrumentos utilizados en su tratamiento, o bien ser pacientes colonizados o infectados los posibles reservorios y que igualmente difundieron el agente a trayés de las manos del personal. ${ }^{5}$

La determinación del patrón de susceptibilidad a los antibióticos, ya sea por el método de difusión en agar o determinación del CIM, es un muy buen método como marcador epidemiológico. ${ }^{13}$ Junto con los marcadores mencionados, la infección intrahospitalaria se caracteriza por la detección de la fuente de contagio y por la observación de la adquisición de la infección en la Unidad y no al ingreso, ${ }^{23}$ hechos que están en concordancia con nuestros hallazgos.

Estos antecedentes nos hacen plantear que estamos en presencia de un brote epidémico de infección intrahospitalaria a Klebsiella pneumoniae multirresistente a antibióticos. Para la confirmación absoluta de la infección intrahospitalaria por este agente es necesario una serotipificación capsular, una tipificación por bacteriocinas, y fagotipificación, lo que permite precisar una relación epidemiológica entre las cepas, ya que uno solo de estos parámetros de relación actualmente no basta. ${ }^{22}$
Entre los antecedentes que presentaron los R.N. que compusieron nuestro grupo estudiado, destaca que el $65 \%$ de ellos fueron varones $y$ un 47\% menores de 37 sem EG, concondando con publicaciones anteriores (Alojipan-Davies).

Sin embargo, otro antecedente que es necesario mencionar es el compromiso hipóxico y fo respiratorio (65\%) que presentaron estos niños (Tabla 3). No nos atrevemos a hacer una relación causa-efecto, sino que deseamos llamar la atención sobre este punto.

Es necesario enfatizar que el cuadro clínico de los R.N. siempre fue descrito como de inicio rápido (horas). Con la aparición de todos los síntomas y signos desde un comienzo. Estos sintomas y signos clínicos que apoyaron el diagnóstico fueron de compromiso gastrointestinal (hepatomegalia, ictericia, esplenomegalia, vómitos), respiratorio (apnea, polipnea, signos de bronconeumonia) y generales (hipoactividad, irritabilidad, fiebre, hiporreflexia, deshidratación), en orden de frecuencia de aparición (Tabla 4).26

Las cepas aisladas no sólo presentaron múltiple resistencia a antibióticos, sino que tenían una gran virulencia, puesto que hubo un $41 \%$ de niños fallecidos, aunque fueron tratados oportunamente con el antibiótico útil, sulfato de colistín, de acuerdo a su antibiograma.

El estudio científico y ordenado de estas sepsis neonatales llevó al acúmulo de datos aqui presentados, los que junto al establecimiento de medidas de vigilancia, saneamiento ambiental, la reparación del lavamanos contaminado, la aplicación de estrictas medidas de asepsia, antisepsia y desinfección, sumado a la educación del personal, permitió el corte del grave brote de infección intrahospitalaria.

\section{RESUMEN}

1. Se estudiaron 17 recién nacidos desde el punto de vista clínico y bacteriológico, que presentaban signos clínicos de sepsis entre abril y septiembre de 1978, y cuyo agente aislado en todos los casos fue Klebsiella pneumoniae.

2. EI cuadro clínico se inició en promedio a los 7.5 días de vida. Cuatro de los recién nacidos tenían antecedentes de rotura prematura de membranas. No hubo relación entre las infecciones del embarazo y la sepsis del recién nacido. El 53\% nacieron por cesárea.

3. E153\% fue pretémino, el $70 \%$ con menos de 
$2.500 \mathrm{~g}$ y el $47 \%$ con scores de Apgar al minuto igual o menor de 5 .

4. El cuadro clínico se manifestó en el $47 \%$ con hepatomegalia, el $70 \%$ con palidez terrosa, el $53 \%$ hipoactividad, el $41 \%$ con deshidratación e ictericia, el $35 \%$ con apnea.

5. Se aislo Klebsiella pneumoniae en personal y ambiente.

6. A todas las cepas se les practicó estudio de sensibilidad a los antibióticos, encontrándose 3 grupos claramente definidos.

7. La mortalidad fue de $41 \%$.

\section{REFERENCLAS}

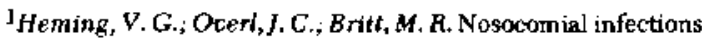
in a Newborn intensive-care unit. New England J. Med. 294: $1310,1976$.

${ }^{2}$ Coldman, D. A. Lecalir, J.; Macone, A. Becterial colonization of ceonates admitted to an istensive care environment. J. Ped. 93: $288,1978$.

${ }^{3}$ Knittle, M. A.i Eitzman, D. V.; Baer, H. Role of hand contamination of personnel in the epidemiology of gram negative nosocomial infection. J. Ped. 86: 433, 1975.

${ }^{4}$ Burke, J. P.; Ingall, D.; Klein, J, O.; Gezan, H. M.; Finlan, $M$. Proteus mirabilis infections in a hospital traced to a buman carrier. New England J. Med. 284: 115, 1971.

${ }^{5}$ Caseurell, M.; Phillips, J. Hands as route of transmission for Klebsiella species. Brit. Med. J. 2: 1301, 1977.

${ }^{5}$ Martin, G.; Myers, M. D. Longitudinal evaluation of neonatal nosocomial infections association of infection with a blood pressure cuff. Pediat. 61:42, 1978.

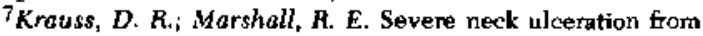
CPAP head box. J. Ped. 86: 286, 1975.

BWeinstein, R. A.; Stamm, W. E.; Kromer, L.; Corey, L. Pressure monitoring devices Overiaoked source of nosocomial infection. JAMA 236: 936, 1976.

${ }^{9}$ Lilien, L. B.; Hartis, V. J,; Ramamurthy, R. S.; Pildes, f. S. Neonatal osteomyelitis of the calcaneus complication of heel punture. J. Ped. 88; 478, 1976.

10 Adler, J. L.; Shulman. J. A.; Terry, P. M.; Feldman, S. B.; Skaliy, $P$. Nosocomial colonization with kamamycin resistant Klebsiella pneumoniae types 2 and $I 1$ in a premature nursery. J. Ped. $77: 376,1970$.

UHill, H. R.; Hunt, C. E.; Matsen.J.M. Nosocomial colonization with Klebsiella type 26 in neonatal intensive care unit associated with an outbreak of sepsis, meningitis and necrotizing enterocolitis. J. Ped. 85: 415, 1974.

12 Hable, K. A.; Motsen, J. M.; Wheeler, D. J.; Hunt, C. E.; Quic, P. G. Klebsiella type 33 septicemia in a infant intensive-care unit. J. Ped. 60: 920, 1972.

${ }^{13}$ Thiemke, W.A., and Nathan, D.M. Simultaneous nosocomial outhreaks caused by multirresistant Klebsiella poeumoniae types 2 and 30. Clín. Microb. 8: 769, 1978.

14 Salzman, T. C., Ctark, J. J., and Klemm, L. Hand contamination of personnel as mechanism of cross-infection in nosocomial infections with antibiotic resistant Escherichía coli and Klebsiella aerobacter. Antímicrob. Agents Chemother, 7: 97, 1967.

15 Hinshate, V.; Punk, J.; Allison, M. J.; Dalton, H. P. Frequency of $\mathbf{F}$ factor mediated multiple drug resistence in Klebsiella and Aerobacter. App. Microb. 17: 214, 1969.

16 Gardner, $P$. Studies on the epidemiology of resistance factor. I. Analysis of Klebsiella isolated in general hospital. II. A prospective study of $\mathrm{H}$ factor transfer in the host. Ann. Intern. Med. f1: 1, 1969.

${ }^{17}$ Lennette, E. H.; Spatudling, E. H., and Truard, J. P., Editors. Manual of clinical Micnobiology. Washington, D.C., 1974. American Society for Microbiology.

${ }^{18}$ Edtuards, P. R., and Exing, W. H. Identification of Enterobac teriaceae. 3rd Burgess Publishing Co. Minneapolis, 1979.

${ }^{19}$ Baver, A. W, Kirby, W. N.; Sherrts, J. C.; Tuck, M. Antibiotic susceptibility testing by standardizing single disc method. Am. J. Clin. Path. 45: 493, 1966.

${ }^{20}$ Daxis, T. J., and Matsen, J. M. Prevalence and characterlstic of Klebsiella species: relation to association with hospital environment. J. Inf. Dis. 130: 402, 1974.

2l Sprumt, K.; Redman, W, Evidence suggesting importance of role of interbacterial inhibition in maintaining balance of nomal flora. Ann. Int. Med. 68: 579, 1968.

22Rennie, R. P.; Nord, C. E.; Joberg, L. S., and Duncan, l. B. A. Comparison of bucteriophage typing, serotyping as aids in epidemiological surveillance of Klebsiella infection. J. Clin. Microb, 8: 638, 1978

23 Feingold, $D, S$. Hospital acquired infections. Now England. J. Med. 283: 1384, 1970 .

${ }^{24}$ Seldon, R.; Lee, S.; Wang, W. Ll,; Bennett, J. V. Nosocomial Klebsiella infections, intestinal colonization as a reservoir. Ann. Intern. Med. 74: 657, 1971.

25 Rennie, R. P., and Duncan, I. B. R. Emergence of gentamicin resistant Klebsiella in a general hospital. Antimicrob. Agents Chemother. 11: 179, 1977.

${ }^{26}$ Alojipan, L. C.; Andrews, B, F. Neonatal sepsis Clinical Pediatrics. 14: 181, 1975.

${ }^{27}$ Dasies, $P$. Bacterial infection in the fehus and newborn. Arch. Dis. Childhood. 46: L, 197̄l. 